\title{
Expression of SUMO associated proteins in the mouse endometrium is regulated by ovarian hormones throughout the estrous cycle
}

\author{
YI LIU $^{1,2^{*}}$, XIAOFANG MA ${ }^{3 *}$, XUHONG CHEN ${ }^{4}$, JINSHENG CHEN $^{1,2}$, \\ LI YUAN $^{4}$, LILI LI ${ }^{5}$, YAOWU BAI ${ }^{1,2}$ and XIAOZHI LIU ${ }^{3}$ \\ ${ }^{1}$ North China University of Science and Technology, Tangshan, Hebei 063210; ${ }^{2}$ Department of Anesthesiology, \\ Tangshan Maternity and Child Health Care Hospital, Tangshan, Hebei 063000; ${ }^{3}$ Central Laboratory; \\ ${ }^{4}$ Department of Gynecology and Obstetrics, The Fifth Central Hospital of Tianjin, Tianjin 300450; \\ ${ }^{5}$ Key Laboratory of Cancer Prevention and Therapy, Department of Bone and Soft Tissue Tumors, \\ National Clinical Research Center for Cancer, Tianjin Medical University Cancer Institute and Hospital, \\ Tianjin 300060, P.R. China
}

Received January 12, 2019; Accepted November1, 2019

DOI: $10.3892 /$ etm. 2020.8416

\begin{abstract}
The modification of proteins by small ubiquitin-like modifier (SUMO), known as SUMOylation, regulates biological function by changing protein transcription and translation. During the estrous cycle the endometrium undergoes continual change to processes including cell proliferation, secretion and exfoliation and these changes are regulated by the levels of ovarian hormones. Increasing the expression of SUMO family members has previously been shown to promote proliferation and invasion of endometrial cancer cells. However, limited research has been carried out into the expression and function of SUMO in the mammalian endometrium. In the present study, the level and localization of SUMO-associated proteins throughout the natural estrous cycle in the mouse uterus was determined using immunohistochemical staining and western blot analysis. The association between the spatiotemporal expression of these SUMO modified proteins and SENPs in endometrium and the concentration of ovarian hormones during estrous cycle was revealed. The present study clarified
\end{abstract}

Correspondence to: Dr Xiaozhi Liu, Central Laboratory, The Fifth Central Hospital of Tianjin, 41 Tanggu Zhejiang Road, Binhai New Area, Tianjin 300450, P.R. China

E-mail:1xz7997@126.com

Dr Yaowu Bai, Department of Anesthesiology, Tangshan Maternity and Child Health Care Hospital, 14 Jianshe South Road, Tangshan, Hebei 063000, P.R. China

E-mail: bywyjh@163.com

*Contributed equally

Key words: estrous cycle, endometrium, small ubiquitin-like modifier, sentrin specific protease, small ubiquitin-like modifier-conjugating enzyme UBC9 the role of SUMOylation in maintenance of normal estrous cycling and may have important significance in the study of hormone-dependent endometrial diseases.

\section{Introduction}

Small ubiquitin-like modifier (SUMO) modification (SUMOylation) is a highly dynamic and reversible post-translational modification of proteins that has been implicated in various biological functions, including gene transcriptional regulation, protein expression and interaction, cell proliferation and apoptosis $(1,2)$. The SUMO family consists of five isoforms and in vertebrates, there are three genes encoding distinct SUMO proteins, SUMO1, SUMO2 and SUMO3 (3). The SUMO2 and SUMO3 proteins share a high degree of homology and are usually considered together as SUMO2/3 (2). SUMOylation involves a series of different proteins and enzymes (4), including SUMO activating enzyme subunits 1 and 2, SUMO-conjugating enzyme UBC9 (UBC9) and SUMO protein ligases, including the E3 protein ligase PIAS family ligases, E3 SUMO-protein ligase RANBP2 and human polycomb group proteins $(5,6)$. The process of dissociating SUMO subunits from target proteins is referred to as de-SUMOlyation, and is carried out by sentrin specific proteases (SENPs). Six SENP family proteins have been identified in mammals (SENP1, SENP2, SENP3, SENP5, SENP6 and SENP7) and each has a different subcellular localization and substrate specificity and participates in different physiological and pathological processes $(7,8)$. All of these SUMO-associated proteins play a key role in reversible SUMO regulation in cells.

The human menstrual cycle lasts approximately 28 days and is under the strict control of estrogen and progesterone, the release of which is regulated by the hypothalamic-pituitary-ovarian axis (9). During the menstrual cycle, the uterus endometrium undergoes a series of morphological and functional changes, cyclically preparing for implantation of a fertilized egg (10). Uterine expression of SUMO isoforms 
and their targets has been described in several endometrial diseases (11) and has been linked with hormone-related endometrial cancers. SUMOylation may play a role in cancer cell proliferation, DNA damage responses, metastasis and apoptosis $(1,12)$. Research has shown that reducing SUMO1 gene expression may inhibit the proliferation and induce apoptosis of Ishikawa cells (13).

Estrogen receptor (ER)- $\beta$ has been identified as a SUMO1 target (14). ER- $\alpha$ has also been reported to undergo SUMO modification, which led to transcriptional activation of the receptor (15). A number of uterine-related diseases, such as endometrial adenocarcinoma (16), endometriosis (17) and cervical high-grade squamous intraepithelial lesion (17), are closely related to the expression of estrogen progesterone and their receptors and it is hypothesized that SUMO regulation is involved in the development and progression of these diseases. However, sex hormone levels are not stable throughout the physiological estrous cycle, so the expression and localization of SUMO-associated proteins in the uterus at various stages of the physiological estrous cycle is of interest.

Rodent estrous cycles are similar to human menstrual cycles (18). The mouse estrous cycle typically lasts 4-5 days (19). Vaginal smear cytology can be used to identify the five stages of the estrous cycle: Proestrus, estrus, metestrus I, metestrus II and diestrus (20). In the present study, the stages of the estrous cycles of female C57BL/6 mice were determined based on the morphology of exfoliated vaginal cells, and the levels of estrogen and progesterone were measured at different periods of the estrous cycle. Changes in the endometrium at the different periods were observed using hematoxylin and eosin $(\mathrm{H} \& \mathrm{E})$ staining. Immunohistochemistry (IHC) was used to determine the localization and expression of SUMO-associated proteins in the mouse endometrium throughout the estrous cycle. Protein expression was also analyzed by western blotting.

The purpose of the present study was to assess changes in SUMO-associated protein expression and localization throughout the endometrium during different stages of the estrous cycle, which exhibit different ovarian hormone levels. Investigating the role of SUMO-associated proteins in the maintenance of the normal estrous cycle may elucidate their role in hormone-dependent endometrial disease.

\section{Materials and methods}

Experimental animals. A total of 40 C57BL/6 female mice (age, 6-8 weeks, weight, $20 \pm 2 \mathrm{~g}$ ) were purchased from the Animal Center of the Institute of Cancer Research, Chinese Academy of Medical Sciences. Animals were maintained under specific pathogen-free conditions at the Animal Experimental Center of the Fifth Central Hospital of Tianjin (Tianjin, China) at $20-25^{\circ} \mathrm{C}$ with $50 \pm 5 \%$ humidity and on a $12 \mathrm{~h}$ light/dark cycle. Animals had free access to food and water. All surgical procedures were performed as approved by the Principles of Laboratory Animal Care (21) and according to the Ethics Committee of The Fifth Central Hospital of Tianjin.

Papanicolaou (Pap) stain. The tip of a sterile cotton swab moistened with saline was placed in the mouse vagina, gently rotated and the collected vaginal mucus then smeared onto a slide and dried naturally. Pap Stain (Beijing Solarbio
Science \& Technology Co.,Ltd.) was used for staining according to the manufacturer's instructions. The smears were evaluated using a light microscope (DP73; Olympus Corporation) under x10 and x40 objective lenses. Vaginal smear staining was used to predict estrous cycle stage using the ratio of the three cell types observed: Round and nucleated cells were epithelial cells, irregular cells without nuclei were cornified cells and the small round cells were leukocytes. The mouse complete estrus cycle lasts for 4-5 days and can be divided into five phases. The proestrus stage has a predominance of anterior keratinocyte cells. The estrus stage is characterized by a large number of non-nucleated keratinized squamous cells, which appear in clusters. The metestrus I stage has reduced squamous epithelial cells compared to other stages with nuclear epithelium and/or keratinization and the appearance of leucocytes. The metestrus II stage has a predominance of leucocytes. The diestrus stage consists predominantly of nuclear epithelial cells, and occasional leucocytes and keratinocytes (20).

Sample collection. Mice were anesthetized by isoflurane inhalation, and blood samples were collected from the ophthalmic vein at different estrous cycle stages ( $n=3 /$ group). Blood samples were incubated for $1 \mathrm{~h}$ at $4^{\circ} \mathrm{C}$ and then centrifuged at $1,000 \times \mathrm{g}$ for $10 \mathrm{~min}$ at $4^{\circ} \mathrm{C}$. Serum was collected and stored at $-80^{\circ} \mathrm{C}$ until further analysis. Anesthetized mice were sacrificed by cervical dislocation and uterine tissues were collected, with one half thoroughly washed with phosphate buffered saline and frozen at $-80^{\circ} \mathrm{C}$, and the other half placed in an embedding box and fixed with $4 \%$ paraformaldehyde for $24 \mathrm{~h}$ at $4^{\circ} \mathrm{C}$. Fixed tissues were dehydrated and immersed in xylene and then embedded in paraffin wax and sectioned into a thin ribbon of $6-\mu \mathrm{m}$ thickness for $\mathrm{H} \& \mathrm{E}$ and IHC staining.

Enzyme-linked immunosorbent assay (ELISA). The serum of each mouse was divided according to the estrous cycle stage, and concentrations of serum pituitary hormones [luteinizing hormone (LH; cat. no. XYA441Mu; BioLegend, Inc.) and follicle stimulating hormone (FSH; cat. no. EF013606; LSM Bio)] and ovarian hormones [estradiol (E2; cat. no. KGE014; R\&D Systems, Inc.) and progesterone (Pr; cat. no. ab108670; Abcam)] were quantified by ELISA kits according to the manufacturer's instructions.

$H \& E$ stain and IHC staining. Mouse uterus paraffin sections from different estrous cycle stages were stained with $\mathrm{H} \& \mathrm{E}$ according to the kit instructions (cat. no. G1120; Beijing Solarbio Science \& Technology Co., Ltd.). IHC staining was performed using a universal IHC kit (mouse/rabbit polymer assay system; cat. no. PV-6000; OriGene Technologies, Inc.) according to the manufacturer's instructions. The antibodies used were supplied by Abcam and dilutions were as follows: SUMO1 (cat. no. ab133352; 1:200), SUMO2/3 (cat. no. ab3742; 1:300), UBC9 (cat. no. ab7585; 1:500), SENP1 (cat. no. ab108981, 1:300), SENP2 (cat. no. ab58418, 1:500), SENP3 (cat. no. ab71677, 1:500) and SENP5 (cat. no. ab583477, 1:200). Stained images were obtained using light microscopy (DP73 microscope; Olympus Corporation). Yellow or brown-yellow granules in the cytoplasm and/or the nucleus indicated positive staining. Two pathologists, blind to the origin of the slides, observed images independently. Ten 
high power microscopic fields (x400) were selected in a homogeneous staining area and the level of positive staining was determined using two methods. The first method evaluated the numbers of stained cells. If no stained cells were seen the field of view was rated 0 , if $<25 \%$ of cells were stained the field of view was rated 1 , if $26-50 \%$ of cells were stained the field of view was rated 2 and if $\geq 51 \%$ of cells in the field of view were stained it was rated 3 . The second method evaluated the staining intensity. No staining was rated 0 , pale yellow staining was rated 1, dark yellow staining was rated 2 and brown was rated 3. The scores obtained from both methods were then added to produce the final staining evaluation. If a field of view was rated 0-1 it was considered not to express the protein of interest (-), if it was rated 2 there was considered to be a low level of protein expression (+), a rating of 3-4 was considered moderate expression $(++)$ and a rating of 5-6 was considered a high level of protein expression (+++). The uterine basal lamina could be distinguished from the functional uterine layers. The endometrium basal layer made up the inner third of the uterus, close to the myometrium and was not affected by hormones so underwent no cyclical changes. The functional layer made up the outer two thirds, close to the uterine cavity and was periodically affected by hormones, this could be divided into the dense layer and the sponge layer.

Western blot analysis. To avoid de-SUMOylation of proteins, the uterine tissues were homogenized in pre-chilled RIPA lysis buffer (EMD Millipore) containing $20 \mathrm{mM}$ N-ethylmaleimide and $1 \mathrm{mM}$ PSMF (Beijing Solarbio Science \& Technology Co., Ltd.). Tissue lysates were centrifuged at $10,778 \mathrm{x}$ g for $15 \mathrm{~min}$ at $4^{\circ} \mathrm{C}$ (1730 GENESPEED). Supernatants were collected and protein concentrations measured. Samples were mixed with sample buffer (Invitrogen; Thermo Fisher Scientific, Inc.) and incubated at $70^{\circ} \mathrm{C}$ for $10 \mathrm{~min}$. Samples $(30 \mu \mathrm{g})$ were then resolved by SDS-PAGE (4-15\% gels; Invitrogen; Thermo Fisher Scientific, Inc.), and then transferred onto a PVDF membrane (EMD Millipore). The membrane was blocked in a Tris-HCl-buffered salt solution containing 0.1\% Tween-20 (TBST) and 5\% skimmed milk powder at room temperature for $1 \mathrm{~h}$, then incubated with primary antibodies provided by Abcam overnight at $4^{\circ} \mathrm{C}$ in PBS with $0.1 \%$ Tween-20 and $5 \%$ skimmed milk powder (Thermo Fisher Scientific, Inc.), at the following dilutions: Anti-SUMO1 (cat. no. ab11672; 1:1,000), anti-SUMO2/3 (cat. no. ab3742; 1:1,000), anti-UBC9 (cat. no. ab7585; 1:1,000), anti-SENP1 (cat. no. ab108981; 1:1,000), anti-SENP2 (cat. no. ab58418; 1:1,000), anti-SENP3 (cat. no. ab71677; 1:800), anti-SENP5 (cat. no. ab583477; 1:500). Membranes were then incubated with secondary peroxidase-conjugated goat anti-rabbit IgG (cat. no. 111-035-003; 1:2,000; Jackson ImmunoResearch Laboratories, Inc.) at room temperature for $2 \mathrm{~h}$. Membranes were incubated with Pierce ${ }^{\mathrm{TM}}$ Fast Western Blot kit ECL reagent (cat. no. 35055; Thermo Fisher Scientific, Inc.) for $5 \mathrm{~min}$ and then protein signals were detected using a C-Digit Blot Scanner (LI-COR Biosciences), ImageJ (version 1.48, National Institutes of Health) was used to quantify protein bands on X-ray film.

Statistical analysis. All data are presented as the mean \pm SD of three independent experiments. Western blots were quantified using GraphPad Prism version 5.0 (GraphPad Software,
Inc.), and statistical analysis was performed using SPSS 17.0 (SPSS, Inc.). Student's t-test or one-way analysis of variance followed by Dunnett's test was performed. $\mathrm{P}<0.05$ was considered a statistically significant difference.

\section{Results}

Vaginal smear cytology. Typical vaginal smear cytology was seen in 35 mice in the current study. These mice were classified into five stages of the estrous cycle according to cytological criteria, as described in Table I. The largest proportion (43\%) of mice were in diestrus, while only $9 \%$ of mice were in estrus (Fig. 1A; Table I).

Expression levels of serum hormones during the estrous cycle. Serum FSH levels were $4.41 \pm 0.40 \mathrm{mIU} / \mathrm{ml}$ at proestrus, $2.84 \pm 0.35 \mathrm{mIU} / \mathrm{ml}$ at estrus, $1.72 \pm 0.44 \mathrm{mIU} / \mathrm{ml}$ at metestrus I, $1.51 \pm 0.42 \mathrm{mIU} / \mathrm{ml}$ at metestrus II and $0.75 \pm 0.41 \mathrm{mIU} / \mathrm{ml}$ at diestrus, with a peak in proestrus compared with other periods $(\mathrm{P}<0.05)$. Serum $\mathrm{LH}$ was $4.45 \pm 0.51 \mathrm{mIU} / \mathrm{ml}$ at proestrus, $7.10 \pm 0.41 \mathrm{mIU} / \mathrm{ml}$ at estrus, $4.67 \pm 0.35 \mathrm{mIU} / \mathrm{ml}$ at metestrus I, $6.72 \pm 0.37 \mathrm{mIU} / \mathrm{ml}$ at metestrus II and $10.82 \pm 0.49 \mathrm{mIU} / \mathrm{ml}$ at diestrus, showing a + significant peak at diestrus compared with other periods $(\mathrm{P}<0.05)$. Serum E2 levels were significantly higher $(\mathrm{P}<0.05)$ in estrus $(37.02 \pm 2.53 \mathrm{mIU} / \mathrm{ml})$ than in proestrus $(24.41 \pm 2.73)$, metestrus I $(25.67 \pm 2.86)$ and metestrus II $(27.63 \pm 2.04 \mathrm{mIU} / \mathrm{ml})$ but there was no significant difference compared with diestrus $(27.71 \pm 2.73 \mathrm{mIU} / \mathrm{ml})$. Serum Pr levels were significantly lower $(\mathrm{P}<0.05)$ in estrus $(12.80 \pm 1.44 \mathrm{ng} / \mathrm{m})$ than in proestrus $(21.36 \pm 1.50 \mathrm{ng} / \mathrm{ml})$, metestrus I $(26.23 \pm 1.73 \mathrm{ng} / \mathrm{ml})$, metestrus II $(24.96 \pm 1.45 \mathrm{ng} / \mathrm{ml})$ and diestrus $(25.73 \pm 1.63 \mathrm{ng} / \mathrm{ml})$, but there were no significant differences between other stages of the estrus cycle (Fig. 1B).

$H \& E$ staining. The results of $\mathrm{H} \& \mathrm{E}$ staining showed that in proestrus the endometrial columnar epithelial cells and stromal cells had proliferated, a thickened intimal connective tissue displayed cellular proliferation and the number of blood vessels had increased in comparison with diestrus (Fig. 2). Estrus exhibited endometrial hyperplasia and hyperemia, endometrial edema and bleeding with erythrocyte exudation. Metestrus I displayed degenerated endometrium and capillary micro-bleeding. Metestrus II had further degeneration of endometrial columnar epithelium cells and endometrial atrophy. Until diestrus, the endometrium was very thin with atrophied endometrial matrix, but glands gradually increased with the start of a new estrous cycle.

Murine endometrial expression and localization of SUMO pathway members during the estrous cycle. Uterine IHC was performed to investigate the expression and localization of SUMO-associated proteins in the endometrium during the estrous cycle (Fig. 3). SUMO1 was mainly detected in uterine luminal and glandular epithelial cells of the endometrium, during the ovulation period, with a high level of staining associated with increased columnar epithelium on the intima surface. Unlike SUMO1, SUMO2/3 was mainly detected in the endometrial stroma, with low level staining in columnar epithelium and glands. The expression of SUMO2/3 showed no marked changes during the estrous cycle. UBC9, the only E2 binding enzyme, exhibited extensive 
Table I. Proportion and characteristics of vaginal smears of 35 mice at different stages of the estrous cycle based on Papanicolaou stain.

\begin{tabular}{|c|c|c|c|}
\hline Estrous cycle stage & $\begin{array}{l}\text { Number } \\
\text { of mice }\end{array}$ & $\begin{array}{l}\text { Proportion out of total } \\
\text { number of mice }(\%)\end{array}$ & Main features of vaginal smear \\
\hline Proestrus & 7 & 20 & $\begin{array}{l}\text { Epithelial and stromal cell proliferation. } \\
\text { Predominance of anterior keratinocyte cells }\end{array}$ \\
\hline Estrus & 3 & 9 & $\begin{array}{l}\text { Endometrial hyperplasia, congestion and edema. } \\
\text { Non-nucleated keratinized squamous cells appear in clusters }\end{array}$ \\
\hline Metestrus I & 6 & 17 & $\begin{array}{l}\text { Luteal formation. } \\
\text { Predominance of non-nucleated and keratinized squamous cells }\end{array}$ \\
\hline Metestrus II & 4 & 11 & $\begin{array}{l}\text { Luteal degeneration. } \\
\text { A predominance of leucocytes }\end{array}$ \\
\hline Diestrus & 15 & 43 & $\begin{array}{l}\text { Follicles begin to mature. } \\
\text { Most cells are nuclear epithelium there are occasional } \\
\text { keratinocytes and leucocytes }\end{array}$ \\
\hline
\end{tabular}

A

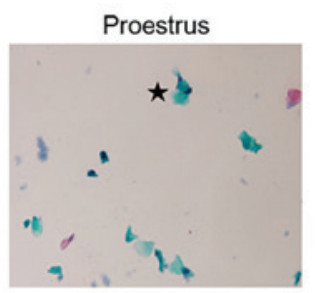

B

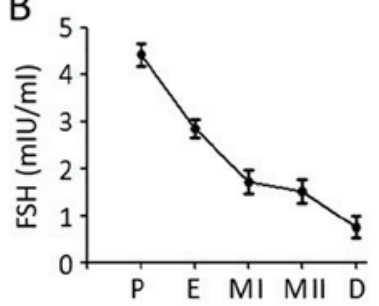

Estrus
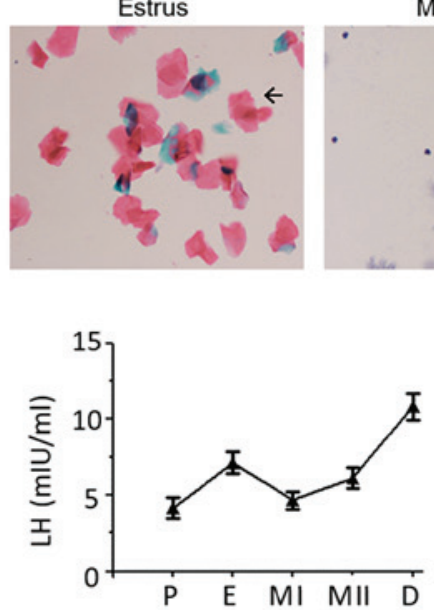

Metestrus I

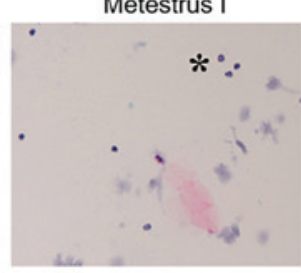

Metestrus II
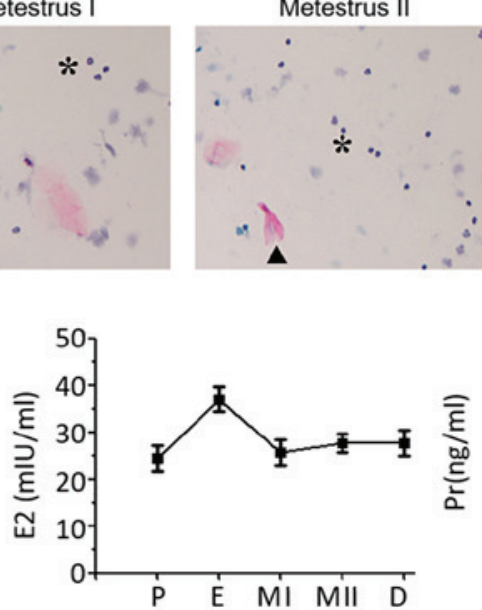

Diestrus
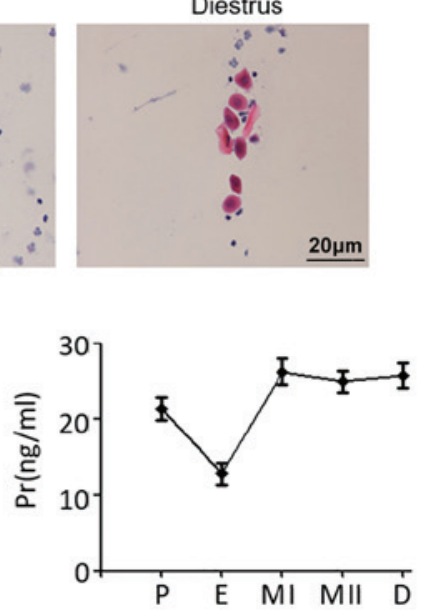

Figure 1. PAP staining of vaginal exfoliated cells and serum hormone levels during different stages of the mouse estrous cycle. (A) Images of Pap-stained vaginal exfoliated cells: Proestrus-anterior keratinocyte cells $(n=7)$; estrus-non-nucleated keratinized squamous cells (n=3); metestrus I-reduced non-nucleated keratinized squamous cells and appearance of leucocytes $(n=6)$; metestrus II-predominance of leucocytes (n=4); diestrus-predominantly nuclear epithelial cells, occasional leucocytes and keratinocytes $(n=15)$. (B) Serum FSH, LH, E2 and Pr levels in female mice at different stages of the estrous cycle. E2, estradiol; FSH, follicle stimulating hormone; LH, luteinizing hormone; Pr, progesterone; $\star$, anterior keratinocyte cell; $\leftarrow$, non-nucleated keratinized squamous cell; \#, nuclear epithelial cell; *, leucocytes; P, proestrus; E, estrus; MI, metestrus I; MII, metestrus II; D, diestrus.

uterine expression throughout the estrous cycle. Subsequently, four important SUMO-specific proteases, SENP1, SENP2, SENP3 and SENP5, were detected and found to display marked differences in distribution and expression. SENP1 displayed the strongest and most widely distributed expression, with a high level of staining in the columnar epithelium and endometrial stroma before and after estrus, and gradually decreasing levels in the endometrial stroma during metestrus and diestrus. SENP2 and SENP3 were mainly distributed in endometrial stroma, and showed no significant changes throughout the estrous cycle. The expression of SENP5 was high in the endometrial epidermis, and increased during estrus and metestrus. Characterization of IHC staining for the SUMO-associated proteins during the estrous cycle is shown in Table II.

Uterine expression levels of SUMO pathway members. Western blot analysis was used to further examine whether periodic changes in the endometrium during the mouse estrous cycle were related to de-SUMOylation. The protein expression levels of SUMO1, SUMO2/3, UBC9, SENP1, SENP2, SENP3 and SENP5 were examined in the mouse uterus at different stages of the estrous cycle.

In the mouse estrous cycle, large molecular weight (>100 kDa) SUMO1-conjugated proteins were abundant during estrus and metestrus I, with no significant difference between the two stages, as shown in Fig. 4A and B. The levels of SUMO2/3-conjugated proteins in metestrus II were decreased compared with estrus and metestrus I, but levels were similar to those at diestrus and proestrus (Fig. 4C and D). UBC9 had a similar expression pattern to SUMO2/3. The expression of SENP1 gradually increased from proestrus to diestrus $(\mathrm{P}<0.05)$, but SENP2 remained stable throughout the estrus cycle. The expression of SENP5 was significantly higher in metestrus I compared with proestrus $(\mathrm{P}<0.05)$. Unlike the 
Table II. Classification of immunohistochemistry staining for SUMO pathway members in uterine tissue during the estrous cycle.

A, Proestrus

\begin{tabular}{lcccccc}
\hline & \multicolumn{1}{c}{ Protein } \\
\cline { 2 - 7 } Area of tissue & SUMO1 & SUMO 2/3 & UBC9 & SENP1 & SENP2 & SENP3 \\
\hline Basal lamina & ++ & \pm & +++ & ++ & - & - \\
Functional layers & ++ & - & + & ++ & ++ & - \\
\hline
\end{tabular}

B, Estrus

\begin{tabular}{lcccccc}
\hline & \multicolumn{9}{c}{ Protein } \\
\cline { 2 - 7 } Area of tissue & SUMO1 & SUMO 2/3 & UBC9 & SENP1 & SENP2 & SENP3 \\
\hline Basal lamina & +++ & - & +++ & +++ & + & - \\
Functional layers & +++ & ++ & +++ & ++ & ++ & ++ \\
\hline
\end{tabular}

C, Metestrus I

Protein

\begin{tabular}{lccccccc}
\cline { 2 - 6 } Area of tissue & SUMO1 & SUMO 2/3 & UBC9 & SENP1 & SENP2 & SENP3 & SENP5 \\
\hline Basal lamina & +++ & - & +++ & +++ & - & - \\
Functional layers & +++ & + & ++ & +++ & +++ & ++ \\
\hline
\end{tabular}

D, Metestrus II

Protein

\begin{tabular}{lccccccc}
\cline { 2 - 6 } Area of tissue & SUMO1 & SUMO 2/3 & UBC9 & SENP1 & SENP2 & SENP3 & SENP5 \\
\hline Basal lamina & ++ & + & ++ & +++ & + & + & +++ \\
Functional layers & ++ & + & + & +++ & + & + & +++ \\
\hline
\end{tabular}

E, Diestrus

Protein

\begin{tabular}{lccccccc}
\cline { 2 - 6 } Area of tissue & SUMO1 & SUMO 2/3 & UBC9 & SENP1 & SENP2 & SENP3 & SENP5 \\
\hline Basal lamina & +++ & - & ++ & +++ & + & \pm \\
Functional layers & +++ & + & + & ++ & ++ & ++ \\
\hline
\end{tabular}

-, no expression; +, no significant expression; +, low-level expression; ++, moderate-level expression; +++, high-level expression.

other SENP members, SENP3 was expressed at low levels during the estrous cycle and showed no significant variation in expression between the five stages (Fig. 4E-H).

\section{Discussion}

In mammalian ovaries, pituitary gonadotrophins cause follicles to produce estrogen, and the luteal body to produce estrogen and progesterone $(22,23)$. Secondary sexual characteristics of women, such as breast development, fat distribution, monthly menstruation, regular ovulation and fetal growth and development in utero after conception, are closely related to the regular ovarian secretion of steroid hormones (24). The uterus is a primary target of estrogen and progesterone and the endometrium, cervix and vagina are all important target organs of estrogen and progesterone, the levels of which change regularly during the menstrual cycle. Studies have shown the high expression of estrogen 

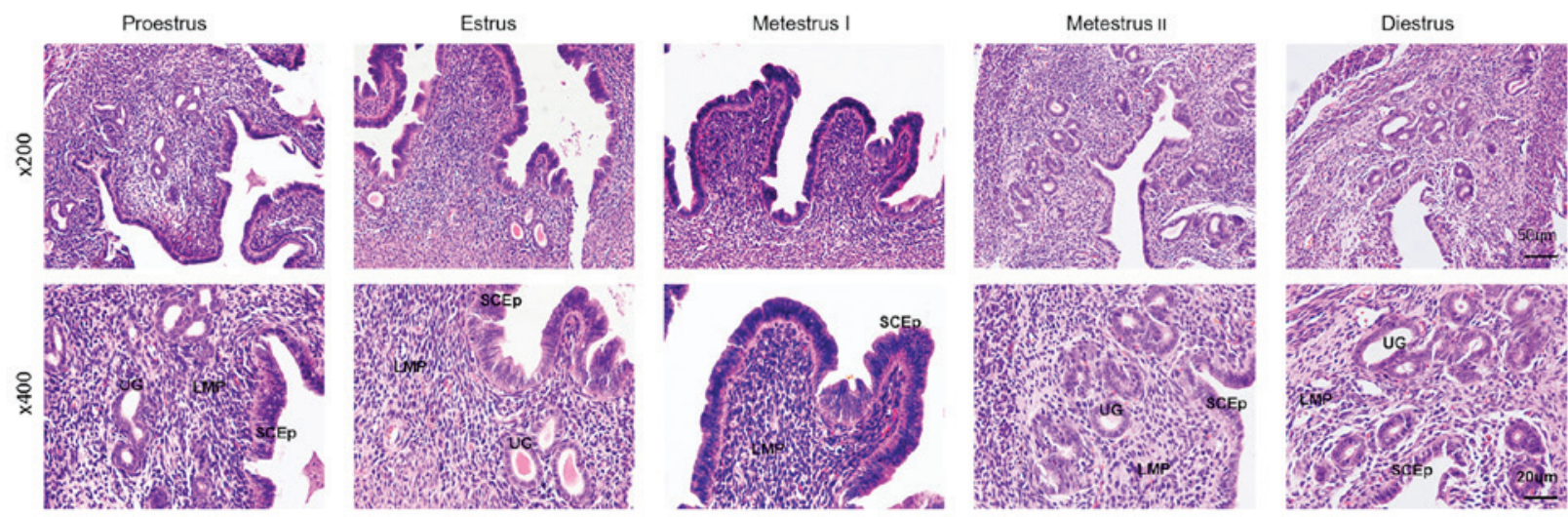

Figure 2. Hematoxylin \& eosin staining of mouse endometrial sections at different stages of the estrous cycle. SCEp, simple columnar epithelium; LPM, lamina propria mucosa; UG, uterine gland.
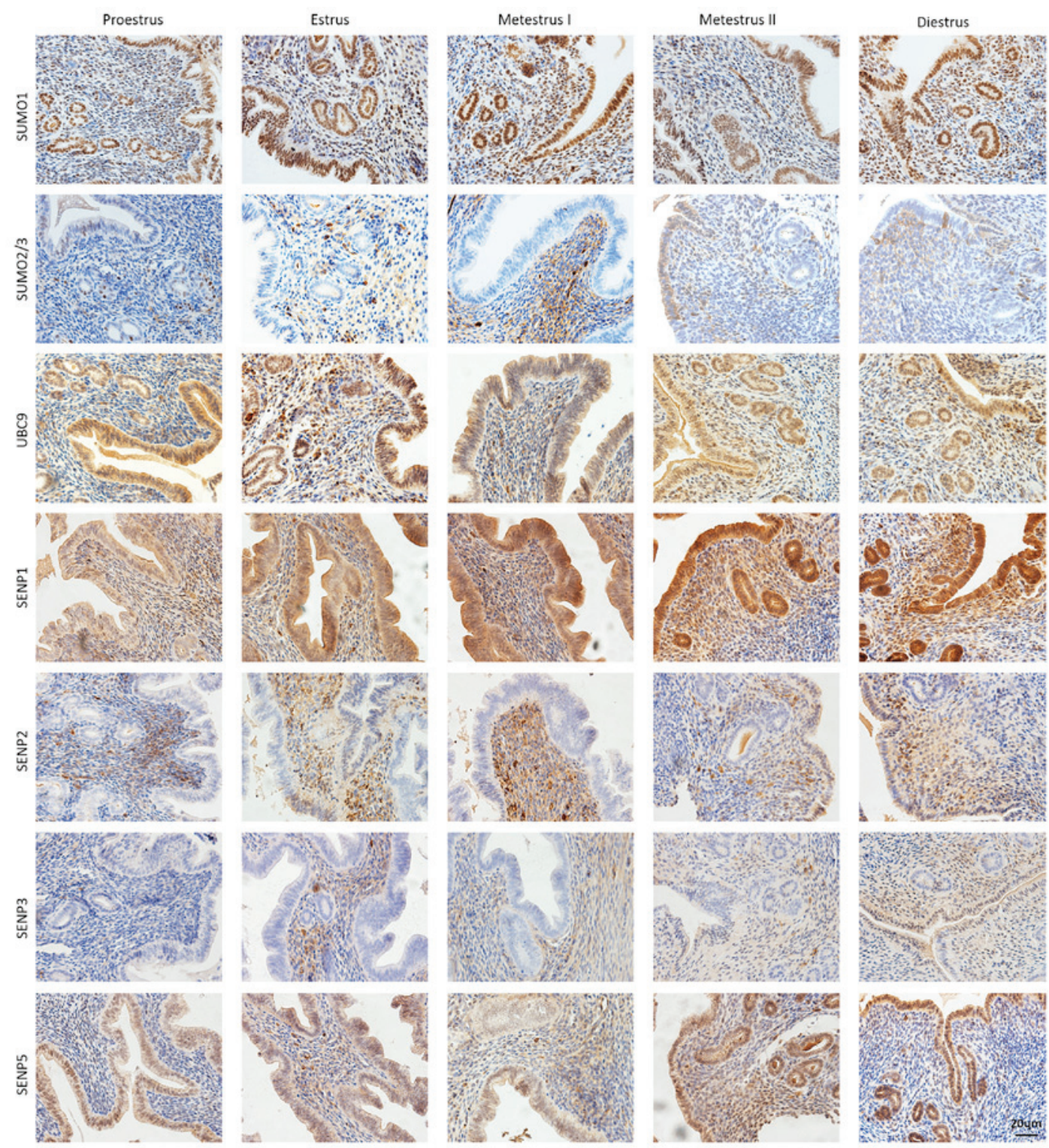

Figure 3. Immunohistochemical staining showing mouse uterine expression of SUMO-related proteins at different stages of the estrous cycle.

and progesterone receptors in endometrial tissue $(25,26)$. In the present study, the vaginal smears of mature female mice were examined and typical cytology patterns were used to characterize different stages in the estrous cycle of the experimental animals (27). Changes in FSH, LH, E2 and Pr levels were detected in mouse serum during the estrous 


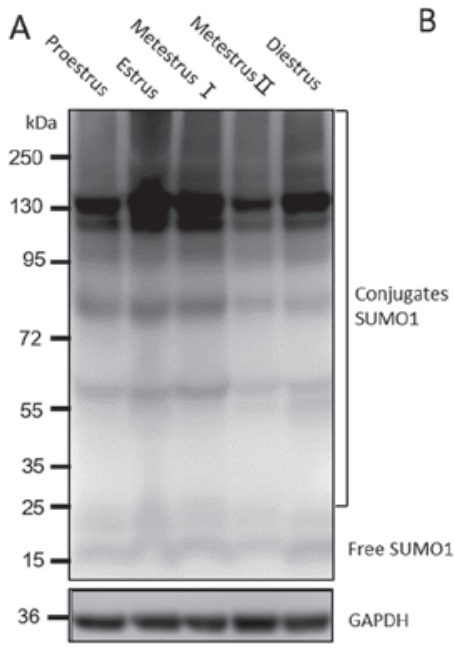

$E$

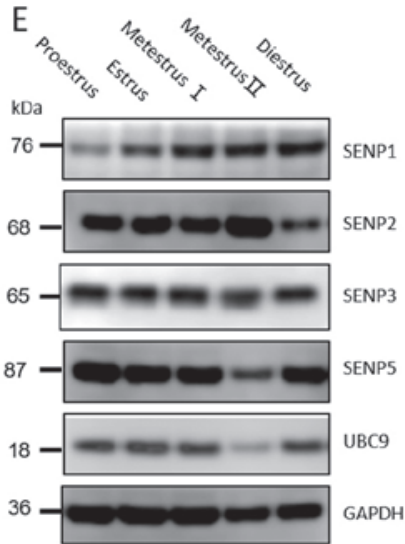

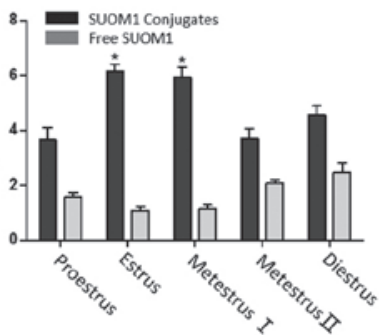

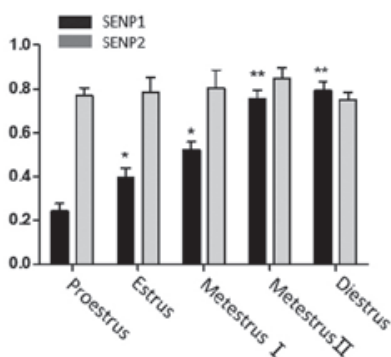

C

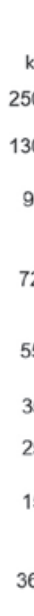

G

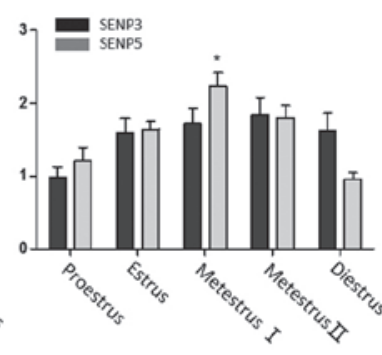

D

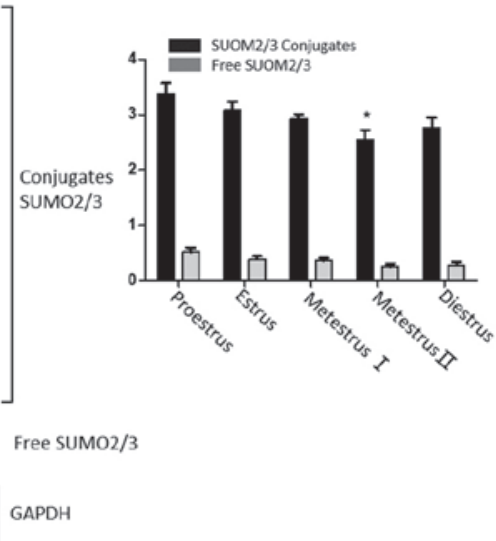

$\mathrm{H}$

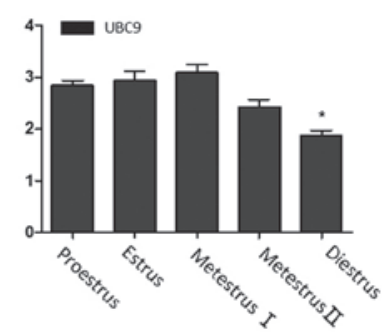

Figure 4. Western blot analysis of SUMO-related proteins in the mouse uterus at different stages of the estrous cycle. (A) Representative western blot of conjugated and free SUMO1 at different stages of the estrous cycle. (B) Normalization of SUMO1 conjugate expression to GAPDH. (C) Representative western blot of SUMO2/3 conjugates at different stages of the estrous cycle. (D) Normalization of SUMO2/3 conjugate expression to GAPDH. (E) Representative western blots of uterine expression of UBC9 and SENP1,2,3 and 5 at different stages of the estrous cycle. GAPDH was used for normalization of the levels of (F) SENP1 and SENP2, (G) SENP3 and SENP5 and (H) UBC9. Data are presented as the mean \pm standard error ( $\mathrm{n}=3) .{ }^{*} \mathrm{P}<0.05,{ }^{* * *} \mathrm{P}<0.01,{ }^{* * * *} \mathrm{P}<0.05$ vs. proestrus. SUMO, small ubiquitin-like modifier; SENP, sentrin specific protease; UBC9, small ubiquitin-like modifier-conjugating enzyme UBC9.

cycle, and endometrium histopathology changes induced by these hormones were observed using H\&E staining. These changes included the regular proliferation and exfoliation of endometrial columnar epithelium and the periodic changes in glands. The above results indicated that changes of serum sex hormones and periodic changes to the endometrium in mice were highly consistent with the equivalent changes of the human menstrual cycle (28).

The endometrium undergoes continual changes in features including cell proliferation, secretion and exfoliation throughout the estrous cycle and these changes are regulated by ovarian hormones (29). A number of studies support a relationship between endogenous hormones and the increased risk of several female cancers. In epidemiologic studies, consistent associations have been observed between risk of breast, ovarian and endometrial cancers and hormonal risk factors caused by high postmenopausal body mass index and postmenopausal hormone use, which suggest the relationship between hormones and gynecological tumors $(26,30)$. Additional studies have confirmed the abnormal expression of SUMO-associated proteins in hormone-dependent gynecological tumors. SUMO1 has an important role in the occurrence and development of certain malignancies, and its expression was shown to be significantly elevated in malignant tumors $(31,32)$. In addition, SUMO1 and ER- $\alpha$ expression were found to be correlated in endometrial carcinoma (13). However, the specific relationship between SUMOs and endometrial cancer is unclear and requires further exploration. To the best of our knowledge, there are few reports on the expression of SUMO isoforms throughout the estrous cycle and the role of SUMO in maintaining the endometrial cycle is unknown. The present study determined the expression and localization of SUMO-associated proteins in the mouse uterus at different stages of the estrous cycle.

Post-translational modifications are a fast and efficient cellular mechanism to react to pathophysiological stimuli $(33,34)$. SUMO-related proteins were largely expressed in a conjugated form. The results of western blot analysis were consistent with IHC data, and both showed cyclical changes in expression patterns and levels of SUMO-related proteins in the endometrium throughout the estrous cycle. It can be hypothesized that SUMO modification is related to hormonal changes and plays an important role in maintaining endometrial function during normal female estrous cycles.

The E2 ligase UCB9 is responsible for the conjugation of SUMO to target molecules (35). The present study found that 
UBC9 was highly expressed throughout the endometrium, with no obvious changes throughout the estrous cycle.

SENPs play a vital role in de-SUMOylation of proteins, and SENP1 was reported to mediate de-SUMOylation during the regulation of cellular proliferation, and involved in the regulation of angiogenesis (36). Increased expression of SENP1 in prostate cancer enhanced AR-dependent transcription, resulting in increased cell proliferation (37). SENP2 expression was reported in bladder cancer, and its overexpression could inhibit the migration and invasion of cultured bladder cancer cells (38). Overexpression of SENP5 in human idiopathic heart failure leads to cardiac dysfunction, accompanied by decreased proliferation of cardiomyocyte and increased apoptosis (39). The present study showed that SENP1, SENP2 and SENP5 were highly expressed throughout the mouse estrous cycle. IHC staining showed that SENP1 was widely distributed and highly expressed in the uterus during the estrous cycle. Western blot analysis showed that SENP5 was highly expressed in the endometrium during estrus. These data suggest that SENP1 and SENP5 are likely to play an important role in regulating deSUMOylation in endometrial tissues, thereby further participating in the proliferation, secretion and exfoliation of uterine cells during the estrous cycle. The results of the present study suggest that SUMO-associated proteins participate in the maintenance of the physiological function of endometrium under the precise regulation of hormones during the estrous cycle. It is hypothesized that hormone level changes, hormone receptor sensitivity increases or any abnormality in SUMO-associated proteins may induce endometrial diseases.

Defining the role of SUMO isoforms in the course of uterine endometrial cyclical changes is likely to be crucial to understand how they function under pathological conditions. However, the mechanism of hormone regulation of target molecules by SUMOylation and de-SUMOylation needs further study. This research is a preliminary study into the relationship between endogenous hormone actions and SUMO expression and how this is associated with uterine tissue remodeling during the menstrual/estrous cycle, and provides the theoretical foundation for pathological studies to screen for potential therapeutic targets of endometrial lesions.

\section{Acknowledgments}

The authors would like to thank Dr Charles Allan for editing the English text of this manuscript.

\section{Funding}

This study was supported by the National Natural Science Foundation of China (grant no. 81471175 and grant no. 81602363) and the Tianjin Binhai New District Health and Family Planning Commission Science and Technology Projects (grant no. 2016BWKY005 and grant no. 2015BWKY005).

\section{Availability of data and materials}

The datasets used and/or analyzed during the current study are available from the corresponding author on reasonable request.

\section{Authors' contributions}

$\mathrm{XL}$ and $\mathrm{YB}$ participated in the design of the study. YL wrote the manuscript. YL, LY, XC, LL and JC collected the tissue samples and participated in the study. YL and XM analyzed and interpreted data. All authors read and approved the final manuscript.

\section{Ethics approval and consent to participate}

All surgical procedures were performed as approved by the Principles of Laboratory Animal Care and according to the Ethics Committee of The Fifth Central Hospital of Tianjin.

\section{Patient consent for publication}

Not applicable.

\section{Competing interests}

The authors declare that they have no competing interests.

\section{References}

1. Han ZJ, Feng YH, Gu BH, Li YM and Chen H: The post-translational modification, SUMOylation, and cancer (Review). Int J Oncol 52: 1081-1094, 2018.

2. Wang YG and Dasso M: SUMOylation and deSUMOylation at a glance. J Cell Sci 122: 4249-4252, 2009.

3. Yeh ET: SUMOylation and De-SUMOylation: Wrestling with life's processes. J Biol Chem 284: 8223-8227, 2009.

4. Hickey CM, Wilson NR and Hochstrasser M: Function and regulation of SUMO proteases. Nat Rev Mol Cell Biol 13: 755-766, 2013.

5. Hochstrasser M: Origin and function of ubiquitin-like proteins. Nature 458: 422-429, 2009.

6. Hay RT: Decoding the SUMO signal. Biochem Soc Trans 41: 463-473, 2013.

7. Wilkinson KA and Henley JM: Mechanisms, regulation and consequences of protein SUMOylation. Biochem J 428: 133-145, 2010.

8. Cimarosti H, Ashikaga E, Jaafari N, Dearden L, Rubin P, Wilkinson KA and Henley JM: Enhanced SUMOylation and SENP-1 protein levels following oxygen and glucose deprivation in neurones. J Cereb Blood Flow Metab 32: 17-22, 2012.

9. Whitcomb BW, Purdue-Smithe A, Hankinson SE, Manson JE, Rosner BA and Bertone-Johnson ER: Menstrual cycle characteristics in adolescence and early adulthood are associated with risk of early natural menopause. J Clin Endocrinol Metab 103: 3909-3918, 2018.

10. Yip KS, Suvorov A, Connerney J, Lodato NJ and Waxman DJ: Changes in mouse uterine transcriptome in estrus and proestrus. Biol Reprod 89: 13, 2013.

11. Flotho A and Melchior F: Sumoylation: A regulatory protein modification in health and disease. Annu Rev Biochem 82: 357-385, 2013.

12. Zheng JD, Liu LL, Wang SF and Huang X: SUMO-1 promotes ishikawa cell proliferation and apoptosis in endometrial cancer by increasing sumoylation of histone H4. Int J Gynecol Cancer 25: 1364-1368, 2015.

13. Su S,Zhang Y and Liu P: Roles of ubiquitination and SUMOylation in DNA damage response. Curr Issues Mol Biol 35: 59-84, 2019.

14. Picard N, Caron V, Bilodeau S, Sanchez M, Mascle X, Aubry M and Tremblay A: Identification of estrogen receptor $\beta$ as a SUMO-1 target reveals a novel phosphorylated sumoylation motif and regulation by glycogen synthase kinase 3 beta. Mol Cell Biol 32: 2709-2721, 2012.

15. Ying SB, Dünnebier T, Si J and Hamann U: Estrogen receptor alpha and nuclear factor $Y$ coordinately regulate the transcription of the SUMO-conjugating UBC9 gene in MCF-7 breast cancer cells. PLoS One 8: e75695, 2013.

16. Guan J, Xie L, Luo X, Yang B, Zhang H, Zhu Q and Chen X: The prognostic significance of estrogen and progesterone receptors in grade I and II endometrioid endometrial adenocarcinoma: Hormone receptors in risk stratification. J Gynecol Oncol 30: e13, 2019. 
17. Vercellini P, Buggio L, Berlanda N, Barbara G, Somigliana E and Bosari S: Estrogen-progestins and progestins for the management of endometriosis. Fertil Steril 106: 1552-1571, 2016.

18. Chaffin CL and Vandevoort CA: Follicle growth, ovulation, and luteal formation in primates and rodents: A comparative perspective. Exp Biol Med (Maywood) 238: 539-548, 2013.

19. Caligioni C: Assessing reproductive status/stages in mice. Curr Protoc Neurosci Appendix 4: Appendix-4I, 2009.

20. Cora MC, Kooistra L and Travlos G: Vaginal cytology of the laboratory rat and mouse: Review and criteria for the staging of the estrous cycle using stained vaginal smears. Toxicol Pathol 43: 776-793, 2015

21. Trappe K, Thomas D, Bikou O, Kelemen K, Lugenbiel P, Voss F Becker R, Katus HA and Bauer A: Suppression of persistent atrial fibrillation by genetic knockdown of caspase 3: A pre-clinical pilot study. Eur Heart J 34: 147-157, 2013.

22. Wood GA, Fata JE, Watson KL and Khokha R: Circulating hormones and estrous stage predict cellular and stromal remodeling in murine uterus. Reproduction 133: 1035-1044, 2007.

23. Chuffa LG, Lupi-Júnior LA, Costa AB, Amorim JP and Seiva FR: The role of sex hormones and steroid receptors on female reproductive cancers. Steroids 118: 93-108, 2017.

24. Annie L, Gurusubramanian G and Roy VK: Estrogen and progesterone dependent expression of visfatin/NAMPT regulates proliferation and apoptosis in mice uterus during estrous cycle. J Steroid Biochem Mol Biol 185: 225-236, 2018.

25. Draper CF, Duisters K, Weger B, Chakrabarti A, Harms AC Brennan L, Hankemeier T, Goulet L, Konz T, Martin FP, et al: Menstrual cycle rhythmicity: Metabolic patterns in healthy women. Sci Rep 8: 14568, 2018.

26. Gao M, Cao C, Zhang X, Tang F, Zhao L, Luo S and Li L: Abnormal expression of estrogen receptor is associated with thin endometrium. Gynecol Endocrinol 35: 544-547, 2019.

27. Cladel NM, Budgeon LR, Balogh KK, Cooper TK, Hu JF and Christensen ND: A novel pre-clinical murine model to study the life cycle and progression of cervical and anal papillomavirus infections. PLoS One 10: e0120128, 2015.

28. Ahrens K, Mumford SL, Schliep KC, Kissell KA, Perkins NJ, Wactawski-Wende J and Schisterman EF: Serum leptin levels and reproductive function during the menstrual cycle. Am J Obstet Gynecol 210: 248.e1-9, 2013.
29. Kalyne B and Bruce DM: Reproductive tract changes during the mouse estrous cycle. Laboratory Equipment, 2014.

30. Brown SB and Hankinson SE: Endogenous estrogens and the risk of breast, endometrial, and ovarian cancers. Steroids 99: 8-10, 2015.

31. Li R, Wei J, Jiang C, Liu D, Deng L, Zhang K and Wang P: Akt SUMOylation regulates cell proliferation and tumorigenesis. Cancer Res 73: 5742-5753, 2013.

32. Liu F, Li L, Li Y, Ma X, Bian X, Liu X, Wang G and Zhang D: Overexpression of SENP1 reduces the stemness capacity of osteosarcoma stem cells and increases their sensitivity to HSVtk/GCV. Int J Oncol 53: 2010-2020, 2018.

33. Rodriguez AC, Blanchard Z, Maurer KA and Gertz J: Estrogen signaling in endometrial cancer: A key oncogenic pathway with several open questions. Horm Cancer 10: 51-63, 2019.

34. Liu A, Zhang D, Yang X and Song Y: Estrogen receptor alpha activates MAPK signaling pathway to promote the development of endometrial cancer. J Cell Biochem 120: 17593-17601, 2019.

35. Hewitt WM, Lountos GT, Zlotkowski K, Dahlhauser SD, Saunders LB, Needle D, Tropea JE, Zhan C, Wei GH, Ma BY, et al: Insights into the allosteric inhibition of the SUMO E2 enzyme Ubc9. Angew Chem Int Ed Engl 5: 5703-5707, 2016.

36. Zhou HJ, Xu Z, Wang ZR, Zhang HF, Simons M and Min W: SUMOylation of VEGFR2 regulates its intracellular trafficking and pathological angiogenesis. Nat Commun 9: 3303, 2018.

37. Cheng JK, Bawa T, Lee P, Gong LM and Yeh E: Role of desumoylation in the development of prostate cancer. Neoplasia 8: 667-676, 2016.

38. Tan MY, Gong H, Wang J, Tao L, Xu DL, Bao E, Liu ZH and Qiu JX: SENP2 regulates MMP13 expression in a bladder cancer cell line through SUMOylation of TBL1/TBLR1. Sci Rep 5: 13996, 2015.

39. Kim EY, Zhang Y, Beketaev I, Segura AM, Yu W, Xi YT, Chang J and Wang J: SENP5, a SUMO isopeptidase, induces apoptosis and cardiomyopathy. J Mol Cell Cardiol 78: 154-164, 2014.

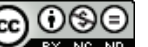

This work is licensed under a Creative Commons Attribution-NonCommercial-NoDerivatives 4.0 International (CC BY-NC-ND 4.0) License. 